\title{
My Escape from the Lab: Scientific Publishing
}

Matteo Cavalleri ${ }^{1}$

${ }^{1}$ Affiliation not available

February 15, 2022

\begin{abstract}
This talk explores roles for postdoctoral STEM researchers in the publishing industry. By sharing my experience I hope to enable a broader understanding of the different roles inside the editorial office of academic journals and the skills required for editorial positions posted by commercial and society publishers.
\end{abstract}

Rich media available at https://speakerdeck.com/teowaits/my-escape-from-the-lab-scientificpublishing

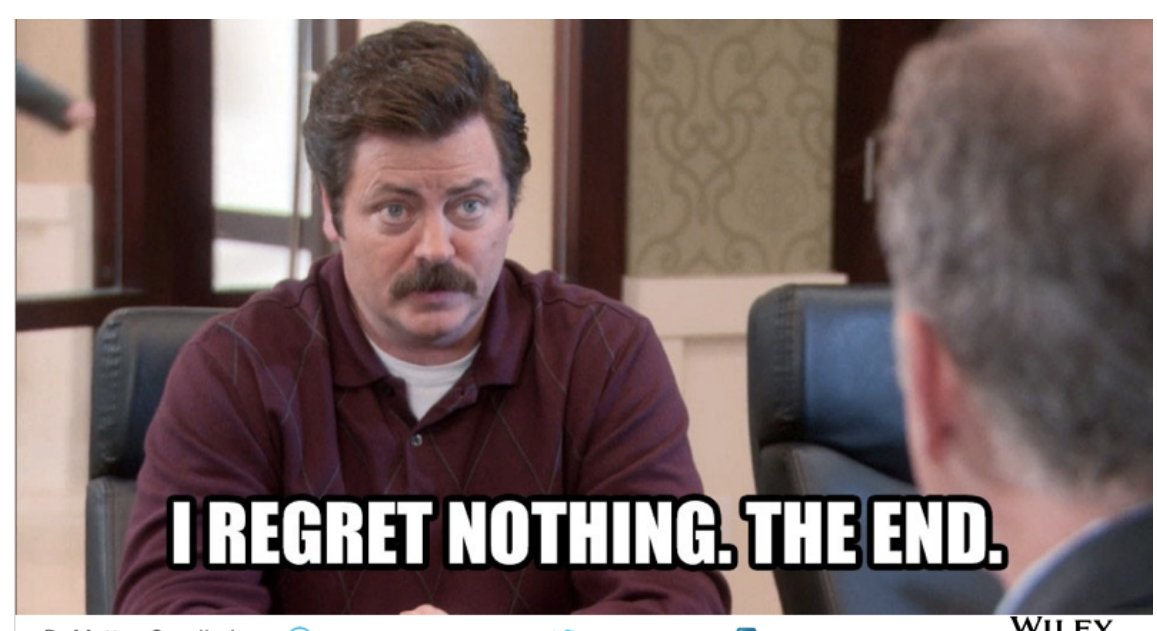

Dr Matteo Cavalleri (mcavalleri@wilev.com @ @phvsicsteo in linkedin.com/teowaits WILEY

Figure 1: Take home message: NO REGRETS 


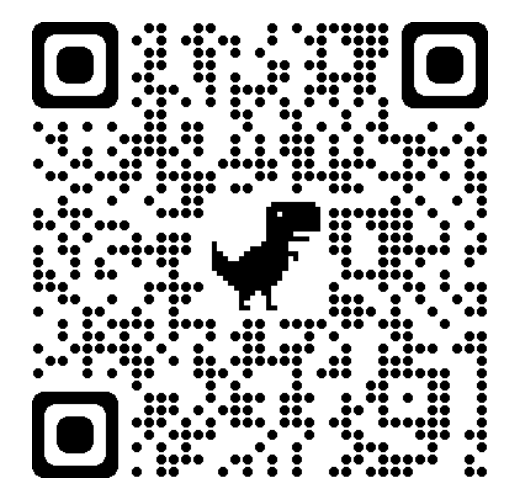

Figure 2: QR CODE TO VIDEO RECORDING OF THE TALK IN 23 SEPT 2020. Otherwise click here. 